\title{
Investigation of a generalized Obukhov Model for Turbulence
}

\author{
A. Baule and R. Friedrich \\ Institute of Theoretical Physics, Westfälische Wilhelms-Universität Münster, \\ Wilhelm-Klemm-Str. 9, 48149 Münster, Germany
}

\begin{abstract}
We introduce a generalization of Obukhov's model [A.M. Obukhov, Adv. Geophys. 6, 113 (1959)] for the description of the joint position-velocity statistics of a single fluid particle in fully developed turbulence. In the presented model the velocity is assumed to undergo a continuous time random walk. This takes into account long time correlations. As a consequence the evolution equation for the joint position-velocity probability distribution is a Fokker-Planck equation with a fractional time derivative. We determine the solution of this equation in the form of an integral transform and derive a relation for arbitrary single time moments. Analytical solutions for the joint probability distribution and its moments are given.
\end{abstract}

PACS numbers: 02.50.-r, 47.27.-i, 05.40.-a, 05.30.Pr

\section{INTRODUCTION}

The statistics of turbulent flows is described by phenomenological theories dating back to Kolmogorov and Obukhov $[1,2,3]$. In a Lagrangian treatment the path $\mathbf{X}(t, \mathbf{y})$ and the velocity $\mathbf{U}(t, \mathbf{y})$ of advected particles initially starting at the position $\mathbf{y}$ are determined by the acceleration $\mathbf{A}(t, \mathbf{y})$ which in principle is given by the Navier-Stokes equation:

$$
\begin{aligned}
& \frac{d \mathbf{X}(t, \mathbf{y})}{d t}=\mathbf{U}(t, \mathbf{y}) \\
& \frac{d \mathbf{U}(t, \mathbf{y})}{d t}=\mathbf{A}(t, \mathbf{y})
\end{aligned}
$$

The central statistical quantity is the joint position-velocity probability distribution of the particle:

$$
f(\mathbf{u}, \mathbf{x}, t)=<\delta(\mathbf{x}-(\mathbf{X}(t, \mathbf{y})-\mathbf{U}(0, \mathbf{y}) t-\mathbf{y})) \delta(\mathbf{u}-(\mathbf{U}(t, \mathbf{y})-\mathbf{U}(0, \mathbf{y}))>\quad,
$$

where the brackets denote a suitable average over a stationary statistical ensemble. Here and in the following we shall be interested in the statistics of increments $\mathbf{u}(t)=\mathbf{U}(t, \mathbf{y})-\mathbf{U}(0, \mathbf{y}), \mathbf{x}(t)=\mathbf{X}(t, \mathbf{y})-\mathbf{U}(0, t) t-\mathbf{y}$. The pdf (2) obeys the initial condition, $f(\mathbf{u}, \mathbf{x}, 0)=\delta(\mathbf{u}) \delta(\mathbf{x})$. Furthermore, Kolmogorov's theory of 1941 (K41) [1] suggests the existence of scaling behaviour

$$
f(\mathbf{u}, \mathbf{x}, t)=\frac{1}{t^{6}} f_{0}\left(\frac{\mathbf{u}}{\sqrt{t}}, \frac{\mathbf{x}}{\sqrt{t}^{3}}\right)
$$

Although such a scaling behaviour may not exist due to intermittency [2] the assumption of normal scaling behaviour (3) usually serves as a first starting point.

Due to the fact that a successfull statistical approach based on a treatment of the Navier-Stokes equation is still missing, one has to resort to heuristic assumptions about the statistics of $\mathbf{A}(t, \mathbf{y})$. An early model for $f(\mathbf{u}, \mathbf{x}, t)$ has been introduced by Obukhov [4], who assumed, that the turbulent acceleration $\mathbf{A}(t, \mathbf{y})$ is a Gaussian, $\delta$-correlated random force:

$$
<A_{i}(t, \mathbf{y}) A_{j}\left(t^{\prime}, \mathbf{y}\right)>=2 \delta_{i j} \delta\left(t-t^{\prime}\right) \quad .
$$

In turn the probability distribution (2) obeys a Fokker-Planck equation:

$$
\frac{\partial}{\partial t} f(\mathbf{u}, \mathbf{x}, t)+\mathbf{u} \cdot \nabla_{\mathbf{x}} f(\mathbf{u}, \mathbf{x}, t)=\Delta_{\mathbf{u}} f(\mathbf{u}, \mathbf{x}, t)
$$

The solution of the Obukhov-model with the initial condition $f(\mathbf{u}, \mathbf{x}, 0)=\delta(\mathbf{x}) \delta(\mathbf{u})$ possess a Gaussian form exhibiting scaling behaviour

$$
<\mathbf{u}^{2}(t)>\sim t \quad, \quad<\mathbf{x}^{2}(t)>\sim t^{3} \quad,
$$

which is consistent with the phenomenological scaling theory of Kolmogorov (K41). However Obukhov's model contradicts recent experimental results [5], [6]: the probability distribution for the velocity increment is far from 
Gaussian and the increment behaviour can not be a simple random walk as suggested by Obukhov. The reason is the intermittent character of turbulent flows. Nonnormal statistics for Lagrangian variables may originate from long time correlations in the turbulent field. During the last few years lots of efforts have been put into the formulation of more sophisticated phenomenological theories which can take into account these facts (c.f. the review article [7]). Recently a connection between the velocity increment statistics of a Lagrangian particle and a type of continuous time random walk has been introduced [8] by a truncation of an infinite chain of evolution equations for multiple particle probability distributions. The following evolution equation for the single time joint position velocity increment probability distribution has been obtained

$$
\begin{aligned}
\left\{\frac{\partial}{\partial t}+\mathbf{u} \cdot \nabla_{x}\right\} f(\mathbf{u}, \mathbf{x}, t) & =\frac{1}{\Gamma(\alpha)} \frac{\partial}{\partial t} \int_{0}^{t} \frac{d t^{\prime}}{\left(t-t^{\prime}\right)^{1-\alpha}} \nabla_{u} Q\left(\mathbf{u}^{2}\right)\left[\nabla_{u} f\left(\mathbf{u}, \mathbf{x}-\mathbf{u}\left(t-t^{\prime}\right), t^{\prime}\right)\right]_{\mathbf{u}^{\prime}=\mathbf{u}}, \\
Q_{i j}\left(\mathbf{u}^{2}\right) & =\mathbf{u}^{2(1-\alpha)}\left[\delta_{i j} Q_{1}+Q_{2} \frac{u_{i} u_{j}}{\mathbf{u}^{2}}\right] .
\end{aligned}
$$

$\left(\Gamma(\alpha)\right.$ denotes the Gamma- function). In the case of isotropic turbulent flows, the matrix $Q_{i j}\left(\mathbf{u}^{2}\right)$ has to be invariant with respect to rotations leading to the form given above. Furthermore, the variables $Q_{1}$ and $Q_{2}$ are constant due to the fact that solutions of the above equation are required to allow for scaling behaviour of the velocity increment $u \approx t^{1 / 2}$ (for constant value of $\alpha$ ). The parameter $\alpha$ is taken from the interval $0<\alpha \leq 1$. This equation generalizes Obukhov's model in several respects. First, it introduces a temporal memory. Second, the simple diffusion process in velocity space is changed to a diffusion process with velocity dependent diffusion coefficient. Third, retardation effects with respect to the spatial coordinate appear. It has been shown that solutions of the resulting equation for the velocity pdf $G(\mathbf{u}, t)=\int d \mathbf{x} f(\mathbf{u}, \mathbf{x}, t)$ yields excellent approximations to the experimentally determined velocity pdf's, provided the parameter $\alpha$ is allowed to vary with the time increment. A complete solution to the equation (7) has not yet been obtained.

In the present paper we investigate a simpler phenomenological model by disregarding the retardation in the spatial coordinate:

$$
\left\{\frac{\partial}{\partial t}+\mathbf{u} \cdot \nabla_{x}\right\} f(\mathbf{u}, \mathbf{x}, t)={ }_{0} D_{t}^{1-\alpha} L\left(\mathbf{u}, \nabla_{u}\right) f(\mathbf{u}, \mathbf{x}, t)+\delta(t) \delta(\mathbf{x}) \delta(\mathbf{u}) \quad .
$$

Here, $L\left(\mathbf{u}, \nabla_{u}\right)$ is a diffusion operator with a velocity dependent diffusion constant (compare eq. (7)). We have explicitly included the initial condition. The operator ${ }_{0} D_{t}^{1-\alpha}$ denotes the Riemann-Liouville fractional differential operator (see e.g. [15]):

$$
{ }_{0} D_{t}^{1-\alpha} F(t)=\frac{1}{\Gamma(\alpha)} \frac{\partial}{\partial t} \int_{0}^{t} \frac{F\left(t^{\prime}\right)}{\left(t-t^{\prime}\right)^{1-\alpha}} d t^{\prime} \quad .
$$

This is a straightforward generalization of integer order differentiation to fractional orders. The presence of the fractional differential operator eq.(9) introduces temporal memory effects depending on the parameter $\alpha$. We assume that $0<\alpha \leq 1$. In the limit $\alpha \rightarrow 1$ our model eq.(8) reduces to the ordinary Obukhov model eq.(5) due to the property ${ }_{0} D_{t}^{0} F(t)=F(t)$ if one takes $L\left(\mathbf{u}, \nabla_{u}\right)$ to be the diffusion operator $L=\Delta_{u}$.

The purpose of the present paper is not to state the accurate evolution equation for the Lagrangian position-velocity increment. This task has to be performed on the basis of a theoretical analysis of the Navier-Stokes equation, as has been started in [8], or by sophisticated data analysis of the experimentally obtained Lagrangian path's of particles. An assessment of the underlying stochastic process, in our opinion, has to include the description of multiple time distributions. Such an analysis for a class of continuous time random walks has recently been started [9]. The purpose of the present paper is to consider an extension of Obukhov's model to a class of fractional diffusion equations, whose study quite seemingly is interesting by its own. It is hoped that the obtained results contribute to a more detailed understanding of Lagrangian turbulence statistics.

The paper is outlined as follows. In the next section we focus on the probability distribution for the velocity increment and review how it can be determined by methods developed in the theory of continuous time random walks (for a recent review of the topic we refer the reader to [10], [1] ). In section III we shall show that the joint position velocity pdf can be expressed as an integral transform

$$
f(\mathbf{u}, \mathbf{x}, t)=\int d \mathbf{z} \int_{0}^{\infty} d s h(\mathbf{z}, \mathbf{x} ; s, t) f_{1}(\mathbf{u}, \mathbf{z}, s)
$$

where $f_{1}(\mathbf{u}, \mathbf{x}, s)$ is the solution of eq. (8) for $\alpha=1$ and $h(\mathbf{z}, \mathbf{x} ; s, t)$ a positive function. Special emphasis is put onto the case of $L\left(\mathbf{u}, \nabla_{u}\right)=\Delta_{u}$. Here, the statistics of the velocity is characterized by subdiffusive behaviour related to temporal memory effects. It is shown that both velocity and position of the particle reveal anomalous diffusive properties. We explicitly determine the probability distributions of velocity and spatial coordinate. For a diffusion operator $L\left(\mathbf{u}, \nabla_{u}\right)$ which allows for scaling solutions $f(\mathbf{u}, \mathbf{x}, s)$ we discuss how normal scaling behaviour $<\mathbf{u}^{2}(t)>\sim t$ may arise in connection with nonnormal statistics. 


\section{THE PROBABILITY DISTRIBUTION OF THE VELOCITY INCREMENT}

After integration over the position variable in eq. (8) an evolution equation for the marginal probability distribution of the velocity $G(\mathbf{u}, t)=\int d \mathbf{x} f(\mathbf{u}, \mathbf{x}, t)$ is obtained in the form of the fractional diffusion equation:

$$
\frac{\partial}{\partial t} G(\mathbf{u}, t)={ }_{0} D_{t}^{1-\alpha} L\left(\mathbf{u}, \nabla_{u}\right) G(\mathbf{u}, t)+\delta(t) \delta(\mathbf{u})
$$

If we take $L\left(\mathbf{u}, \nabla_{u}\right)=\Delta_{u}$ we obtain the fractional diffusion equation for a continuous time random walk [10], [11]. Accordingly the second order moment reveals subdiffusive characteristics: $\left\langle u^{2}(t)>\sim t^{\alpha}\right.$. The corresponding pdf is well-known and will be given below.

The solution of the fractional diffusion equation (11) is conveniently expressed as an integral transform [12], [13]

$$
G(\mathbf{u}, t)=\int_{0}^{\infty} d s h_{\alpha}(s, t) G_{1}(\mathbf{u}, s)
$$

in which the function $G_{1}(\mathbf{u}, s)$ is obtained as solution of the ordinary diffusion equation (11) with $\alpha=1$ and the integral kernel $h_{\alpha}(s, t)$ is given as the single time probability distribution of an inverse Lévy-stable process [12], [13]:

$$
h_{\alpha}(s, t)=\frac{1}{\alpha} \frac{t}{s^{1+1 / \alpha}} L_{\alpha}\left(\frac{t}{s^{1 / \alpha}}\right) \quad .
$$

Here $L_{\alpha}(t)$ denotes a one-sided Lévy-stable distribution of order $\alpha$. This may be shown by inserting the ansatz (12) into eq. (11) leading to the solvability conditions

$$
\begin{aligned}
\frac{\partial}{\partial t} h_{\alpha}(s, t) & =-{ }_{0} D_{t}^{1-\alpha} \frac{\partial}{\partial s} h_{\alpha}(s, t) \\
{ }_{0} D_{t}^{1-\alpha} h_{\alpha}(0, t) & =\delta(t)
\end{aligned}
$$

These two equations determine $h_{\alpha}(s, t)$ in the form eq.(13). The properties of the diffusion operator $L\left(\mathbf{u}, \nabla_{u}\right)$ enter via the pdf $G_{1}(\mathbf{u}, t)$. The form of the pdf, eq. (12) has the following interpretation. There is a usual diffusion process $\mathbf{w}(s)$ described by a Fokker-Planck operator $L\left(\mathbf{w}, \nabla_{w}\right)$ with respect to an intrinsic time $s$. Additionally, there is a prozess $s=s(t)$ relating physical time $\mathrm{t}$ and intrinsic time $\mathrm{s}$ with a probability distribution $h_{\alpha}(s, t)$. The random process $\mathbf{u}(t)$ is given by $\mathbf{u}(t)=\mathbf{w}(s(t))$.

\section{SOLUTION OF THE GENERALIZED OBUKHOV-MODEL}

Motivated by the results for the fractional diffusion equation we look for solutions of eq.(8) which can be expressed as an integral transform similar to eq.(12):

$$
f(\mathbf{u}, \mathbf{x}, t)=\int_{-\infty}^{\infty} d \mathbf{z} \int_{0}^{\infty} d s h(\mathbf{z}, \mathbf{x} ; s, t) f_{1}(\mathbf{u}, \mathbf{z}, s)
$$

$f_{1}(\mathbf{u}, \mathbf{z}, s)$ denotes the solution of the ordinary Obukhov-model eq.(8) specified by $\alpha=1$ :

$$
\frac{\partial}{\partial s} f_{1}(\mathbf{u}, \mathbf{x}, s)+\mathbf{u} \cdot \nabla_{\mathbf{x}} f_{1}(\mathbf{u}, \mathbf{x}, s)=L\left(\mathbf{u}, \nabla_{u}\right) f_{1}(\mathbf{u}, \mathbf{x}, s)
$$

The structure of the pdf, eq. (15), has the following interpretation. Let us consider the case $L=\Delta_{u}$. There is a stochastic process with respect to the intrinsic time $s$ given by

$$
\begin{aligned}
\frac{d \mathbf{z}}{d s} & =\mathbf{u}(s) \\
\frac{d \mathbf{w}}{d s} & =\mathbf{F}(\mathbf{w}, s)
\end{aligned}
$$

Furthermore, there is a process $s(t)$ relating physical time $\mathrm{t}$ and intrinsic time s. The velocity $\mathbf{u}(t)$, thereby, is given by $\mathbf{u}(t)=\mathbf{w}(s(t))$. If the space variable $\mathbf{x}(t)$ would be obtained via $\mathbf{x}(t)=\mathbf{z}(s(t))$ the function $h$ would not depend 
on $\mathbf{x}$ and $\mathbf{z}$ and would be simply given by the function $h_{\alpha}(s, t)$ of the preceding section. However, the space variable $\mathbf{x}(t)$ is defined by the relationship

$$
\frac{d \mathbf{x}(t)}{d t}=\mathbf{u}(t)
$$

This explains the fact why $h(\mathbf{z}, \mathbf{x} ; s, t)$ depends on the two spatial variables $\mathbf{x}, \mathbf{z}$ but not on the velocity $\mathbf{u}$.

In the following we shall derive a fractional differential equation for $h(\mathbf{z}, \mathbf{x} ; s, t)$, corresponding boundary conditions and obtain an explicit solution. We proceed as follows: the ansatz eq.(15) is substituted into the evolution equation (8). The operator $L\left(\mathbf{u}, \nabla_{u}\right)$ only acts on $f_{1}(\mathbf{u}, \mathbf{z}, s)$ such that eq.(16) can be applied:

$$
\begin{aligned}
& \int_{-\infty}^{\infty} d \mathbf{z} \int_{0}^{\infty} d s\left(\frac{\partial}{\partial t}+\mathbf{u} \cdot \nabla_{\mathbf{x}}\right) h(\mathbf{z}, \mathbf{x} ; s, t) f_{1}(\mathbf{u}, \mathbf{z}, s) \\
& ={ }_{0} D_{t}^{1-\alpha} \int_{-\infty}^{\infty} d \mathbf{z} \int_{0}^{\infty} d s h(\mathbf{z}, \mathbf{x} ; s, t)\left(\frac{\partial}{\partial s}+\mathbf{u} \cdot \nabla_{\mathbf{z}}\right) f_{1}(\mathbf{u}, \mathbf{z}, s)+\delta(t) \delta(\mathbf{u}) \delta(\mathbf{x}) .
\end{aligned}
$$

In the integral on the right hand side we perform a partial integration with respect to $\mathbf{z}$ and $s$. Since $f_{1}(\mathbf{u}, \mathbf{z}, s)$ vanishes for $|\mathbf{z}| \rightarrow \infty$ the following boundary terms are obtained:

$$
\left[{ }_{0} D_{t}^{1-\alpha} \int_{-\infty}^{\infty} d \mathbf{z} h(\mathbf{z}, \mathbf{x}, s, t) f_{1}(\mathbf{u}, \mathbf{z}, s)\right]_{s=0}^{s=\infty}
$$

We can assume $h(\mathbf{z}, \mathbf{x} ; s=\infty, t)=0$.

Let us consider the boundary term using the initial condition $f_{1}(\mathbf{u}, \mathbf{z}, s=0)=\delta(\mathbf{u}) \delta(\mathbf{z})$. In that case, the boundary term (20) yields:

$$
\left[{ }_{0} D_{t}^{1-\alpha} \int_{-\infty}^{\infty} d \mathbf{z} h(\mathbf{z}, \mathbf{x} ; s, t) f_{1}(\mathbf{u}, \mathbf{z}, s)\right]_{s=0}^{s=\infty}=-{ }_{0} D_{t}^{1-\alpha} h(0, \mathbf{x} ; 0, t) \delta(\mathbf{u})=-\delta(\mathbf{x}) \delta(\mathbf{u}) \delta(t) \quad .
$$

This term cancels the last term on the right hand side of (19) provided we postulate the validity of the boundary condition

$$
{ }_{0} D_{t}^{1-\alpha} h(\mathbf{z}=0, \mathbf{x} ; s=0, t)=\delta(t) \delta(\mathbf{x}) \quad .
$$

As a consequence, the function $h(\mathbf{z}, \mathbf{x} ; s, t)$ has to obey the following equation

$$
\begin{aligned}
& \left(\frac{\partial}{\partial t}+\mathbf{u} \cdot \nabla_{\mathbf{x}}\right) h(\mathbf{z}, \mathbf{x} ; s, t)=-{ }_{0} D_{t}^{1-\alpha}\left(\frac{\partial}{\partial s}+\mathbf{u} \cdot \nabla_{\mathbf{z}}\right) h(\mathbf{z}, \mathbf{x} ; s, t) \\
& { }_{0} D_{t}^{1-\alpha} h(\mathbf{z}=0, \mathbf{x} ; s=0, t)=\delta(t) \delta(\mathbf{x})
\end{aligned}
$$

This is the generalization of eq. (14).

We have to add the following remarks. First, equation (23) determines the $\mathbf{u}$-independent function $h(\mathbf{z}, \mathbf{x} ; s, t)$ by a relation including the velocity $\mathbf{u}$. The fact that there is no $\mathbf{u}$-dependence has been crucial for arriving at the equations (23). Below, we shall show that we actually can find a u-independent solution. Second, we point out that the properties of the diffusion operator $L\left(\mathbf{u}, \nabla_{u}\right)$ do not show up in the determination of the function $h(\mathbf{z}, \mathbf{x} ; s, t)$. The properties of this operator are included in the pdf $f(\mathbf{u}, \mathbf{z}, s)$ and we have obtained the solution in terms of an integral transform for a large class of stochastic processes.

\section{A. Determination of $h(\mathbf{z}, \mathbf{x} ; s, t)$}

The solution of differential equations containing the Riemann-Liouville fractional differential operator is simplified by changing to Laplace-space. This is due to the fact that the integral in the definition eq.(9) is actually a Laplaceconvolution. Throughout this discussion we denote Laplace-transforms as follows: $\tilde{F}(\lambda):=\mathcal{L}\{F(t)\}:=\int_{0}^{\infty} d t e^{-\lambda t} F(t)$. By performing a Laplace-transformation of eq.(23) we derive the following first order partial differential equation:

$$
\begin{aligned}
\left(\lambda+\mathbf{u} \cdot \nabla_{\mathbf{x}}\right) \tilde{h}(\mathbf{z}, \mathbf{x} ; s, \lambda)-h(\mathbf{z}, \mathbf{x} ; s, 0)= & -\lambda^{1-\alpha}\left(\frac{\partial}{\partial s}+\mathbf{u} \cdot \nabla_{\mathbf{z}}\right) \tilde{h}(\mathbf{z}, \mathbf{x} ; s, \lambda) \\
& +\left(\frac{\partial}{\partial s}+\mathbf{u} \cdot \nabla_{\mathbf{z}}\right)\left[{ }_{0} D_{t}^{1-\alpha} h(\mathbf{z}, \mathbf{x} ; s, t)\right]_{t=0} .
\end{aligned}
$$


The last term on the right hand side is due to the partial time derivative in the fractional differential operator. It can be set to zero: $\left[{ }_{0} D_{t}^{-\alpha} h(\mathbf{z}, \mathbf{x} ; s, t)\right]_{t=0}=0$. Equation (24) is valid for $s>0$. For $s=0$ we have the boundary condition $h(\mathbf{z}, \mathbf{x} ; s, 0)=\delta(\mathbf{z}-\mathbf{x}) \delta(s)$. Thus we obtain the final form of the evolution equation for $\tilde{h}(\mathbf{z}, \mathbf{x} ; s, \lambda)$ :

$$
\left\{\frac{\partial}{\partial s}+\mathbf{u} \cdot \nabla_{\mathbf{z}}+\lambda^{\alpha-1} \mathbf{u} \cdot \nabla_{\mathbf{x}}\right\} \tilde{h}(\mathbf{z}, \mathbf{x} ; s, \lambda)=-\lambda^{\alpha} \tilde{h}(\mathbf{z}, \mathbf{x} ; s, \lambda)
$$

Linear partial differential equations of this type can be solved by the method of characteristics (see e.g. [16]). This method will be applied below.

If we choose $s$ as parameter of the characteristics, eq.(25) can be written as

$$
\begin{aligned}
\frac{d}{d s} \tilde{h}(\mathbf{z}(s), \mathbf{x}(s) ; \lambda, s) & =\left\{\frac{\partial}{\partial s}+\frac{d \mathbf{z}(s)}{d s} \cdot \nabla_{\mathbf{z}}+\frac{d \mathbf{x}(s)}{d s} \cdot \nabla_{\mathbf{x}}\right\} \tilde{h}(\mathbf{z}(s), \mathbf{x}(s) ; \lambda, s) \\
& =-\lambda^{\alpha} \tilde{h}(\mathbf{z}(s), \mathbf{x}(s) ; \lambda, s)
\end{aligned}
$$

Clearly the solution of this differential equation reads

$$
\tilde{h}(\mathbf{z}(s), \mathbf{x}(s) ; \lambda, s)=\tilde{h}(\mathbf{z}(0), \mathbf{x}(0) ; \lambda, 0) e^{-\lambda^{\alpha} s} \quad .
$$

The characteristics $\mathbf{z}(s), \mathbf{x}(s)$ are determined by ordinary first order differential equations:

$$
\begin{array}{lll}
\frac{d \mathbf{z}(s)}{d s}=\mathbf{u} & \rightarrow & \mathbf{z}(s)=\mathbf{u} s+\mathbf{z}_{0} \\
\frac{d \mathbf{x}(s)}{d s}=\lambda^{\alpha-1} \mathbf{u} & \rightarrow & \mathbf{x}(s)=\lambda^{\alpha-1} \mathbf{u} s+\mathbf{x}_{\mathbf{0}}
\end{array}
$$

For the initial condition $\tilde{h}(\mathbf{z}(0), \mathbf{x}(0) ; 0, \lambda)=\tilde{h}\left(\mathbf{z}_{0}, \mathbf{x}_{0} ; 0, \lambda\right)$ we specify a sharp distribution at $\mathbf{x}_{0}$. The dependence on $\lambda$ is chosen as $\lambda^{\alpha-1}$ to be consistent with the boundary condition (23). If we put $\mathbf{z}_{0}=0$ the inital condition takes the form:

$$
\tilde{h}\left(\mathbf{z}_{0}=0, \mathbf{x}_{0} ; 0, \lambda\right)=\delta\left(\mathbf{x}_{0}\right) \lambda^{\alpha-1}=\delta\left(\mathbf{x}-\lambda^{\alpha-1} \mathbf{z}\right) \lambda^{\alpha-1}
$$

Finally we obtain the kernel of the transformation eq.(15) as solution of the partial differential equation (25) in Laplace-space:

$$
\tilde{h}(\mathbf{z}, \mathbf{x} ; s, \lambda)=\delta\left(\mathbf{x}-\lambda^{\alpha-1} \mathbf{z}\right) \lambda^{\alpha-1} e^{-\lambda^{\alpha} s}=\delta\left(\mathbf{x}-\lambda^{\alpha-1} \mathbf{z}\right) \tilde{h}_{\alpha}(s, \lambda)
$$

In the last step we substitute the Laplace-transform of $h_{\alpha}(s, t): \mathcal{L}\left\{h_{\alpha}(s, t)\right\}=\lambda^{\alpha-1} e^{-\lambda^{\alpha} s}$.

From this expression the integral kernel in physical time $h(\mathbf{z}, \mathbf{x} ; s, t)$ is given as inverse Laplace-transform:

$$
h(\mathbf{z}, \mathbf{x} ; s, t)=\mathcal{L}^{-1}\left\{\delta\left(\mathbf{x}-\lambda^{\alpha-1} \mathbf{z}\right)\right\} * h_{\alpha}(s, t)
$$

The asterisk $*$ denotes a Laplace-convolution with respect to $t$. However due to the $\lambda$-dependence of the $\delta$-function a closed form of the inverse transformation $\mathcal{L}^{-1}\left\{\delta\left(\mathbf{x}-\lambda^{\alpha-1} \mathbf{z}\right)\right\}$ could not be calculated and the explicit expression of $h(\mathbf{z}, \mathbf{x} ; s, t)$ remains unknown.

Still the transformation eq.(15) provides a useful equation for characterizing the solution $f(\mathbf{u}, \mathbf{x}, t)$. Since inverse Laplace-transform and integration commute we can give an expression for the transformed solution $\tilde{f}(\mathbf{u}, \mathbf{x}, \lambda):=$ $\mathcal{L}\{f(\mathbf{u}, \mathbf{x}, t)\}$ :

$$
\tilde{f}(\mathbf{u}, \mathbf{x}, \lambda)=\int_{-\infty}^{\infty} d \mathbf{z} \int_{0}^{\infty} d s \tilde{h}(\mathbf{z}, \mathbf{x} ; s, \lambda) f_{1}(\mathbf{u}, \mathbf{z}, s)
$$

Thus statistical quantities can be calculated in Laplace-space and then transformed to physical time. It will become clear in the following that with the help of the integral kernel $\tilde{h}(\mathbf{z}, \mathbf{x} ; s, \lambda)$ the solution of the generalized Obukhovmodel can be completely characterized.

However, several remarks are in order. First of all, one can easily check that the solutions $f(\mathbf{u}, \mathbf{x}, t)$ of eq.(8) are normalized:

$$
\int_{-\infty}^{\infty} d \mathbf{x} \int_{-\infty}^{\infty} d \mathbf{u} f(\mathbf{u}, \mathbf{x}, t)=\int_{-\infty}^{\infty} d \mathbf{u} f(\mathbf{u}, t)=1
$$


Additionally one states that $\tilde{h}(\mathbf{z}, \mathbf{x} ; s, \lambda)$ is always nonegative and so are $h(\mathbf{z}, \mathbf{x} ; s, t), f_{1}(\mathbf{u}, \mathbf{z}, s)$, and, in turn, $f(\mathbf{u}, \mathbf{x}, t)$. This proofs that the generalized Oboukhov model actually defines a probability distribution for the case $0<\alpha<1$.

Second, we have to show that the above assumptions are fullfilled. To this end we note that the quantity $h(\mathbf{z}, \mathbf{x} ; s, t)$ in fact is independent on the velocity $\mathbf{u}$. Furthermore, we have the relationship

$$
\tilde{h}(0, \mathbf{x} ; s, \lambda)=\delta(\mathbf{x}) \tilde{h}_{\alpha}(s, \lambda) \quad,
$$

which in real space reads

$$
{ }_{0} D_{t}^{1-\alpha} h(0, \mathbf{x} ; 0, t)=\delta(\mathbf{x})_{0} D_{t}^{1-\alpha} h_{\alpha}(0, t)=\delta(\mathbf{x})
$$

Since $h_{\alpha}(s, 0)=\delta(s)$ we obtain

$$
h(\mathbf{z}, \mathbf{x} ; s, 0)=\mathcal{L}^{-1}\left\{\delta\left(\mathbf{x}-\lambda^{\alpha-1} \mathbf{z}\right)\right\} * \delta(s) \sim \delta(s)
$$

The case $\alpha=1$ has to lead to the ordinary Obukhov model. Evidently the integral kernel eq. (30) in this limit is given by the Laplace transform

$$
\tilde{h}(\mathbf{z}, \mathbf{x} ; s, \lambda)=\delta(\mathbf{x}-\mathbf{z}) e^{-\lambda s} \quad .
$$

The inverse Laplace-transformation is easily calculated:

$$
h(\mathbf{z}, \mathbf{x} ; s, t)=\delta(\mathbf{x}-\mathbf{z}) \delta(t-s) \quad,
$$

such that the transformation eq.(15) leads to the solution of the ordinary Obukhov-model.

The second limit case is supposed to yield the solution $G(\mathbf{u}, t)$ of the fractional diffusion equation. Performing the integration of eq. (32) with respect to $\mathrm{x}$ results in:

$$
\int_{-\infty}^{\infty} d \mathbf{x} \int_{-\infty}^{\infty} d \mathbf{z} \int_{0}^{\infty} d s \delta\left(\mathbf{x}-\lambda^{\alpha-1} \mathbf{z}\right) \tilde{h}_{\alpha}(s, \lambda) f_{1}(\mathbf{u}, \mathbf{z}, s)=\int_{0}^{\infty} d s \tilde{h}_{\alpha}(s, \lambda) G_{1}(\mathbf{u}, s)
$$

The right hand side of this equation is the Laplace-transform of the solution eq. (12) of the fractional diffusion equation (11). The agreement in this case is achieved due to the choice $\lambda^{\alpha-1}$ in the initial condition eq.(29).

\section{B. The joint probability distribution $f(\mathbf{u}, \mathbf{x}, t)$}

In the following we derive a formal expression for the joint probability distribution $\tilde{h}(\mathbf{z}, \mathbf{x} ; s, \lambda)$. First the Laplacetransform of $f(\mathbf{u}, \mathbf{x}, t)$ is calculated using the transformation eq. (32):

$$
\begin{aligned}
f(\mathbf{u}, \mathbf{x}, \lambda) & =\int_{-\infty}^{\infty} d \mathbf{z} \int_{0}^{\infty} d s \delta\left(\mathbf{x}-\lambda^{\alpha-1} \mathbf{z}\right) \tilde{h}_{\alpha}(s, \lambda) f_{1}(\mathbf{u}, \mathbf{z}, s) \\
& =\int_{0}^{\infty} d s \lambda^{d(1-\alpha)} \tilde{h}_{\alpha}(s, \lambda) f_{1}\left(\mathbf{u}, \lambda^{1-\alpha} \mathbf{x}, s\right)
\end{aligned}
$$

Here the integration with respect to $\mathbf{z}$ is performed in $d$ dimensions. Let us assume that the diffusion operator $L\left(\mathbf{u}, \nabla_{u}\right)$ allows for solutions exhibiting scaling behaviour of the form

$$
f_{1}(\mathbf{u}, \mathbf{z}, s)=\frac{1}{s^{(1+2 \delta) d}} H\left(\frac{\mathbf{z}}{s^{1+\delta}}, \frac{\mathbf{u}}{s^{\delta}}\right)
$$

This yields:

$$
f(\mathbf{u}, \mathbf{x}, \lambda)=\int_{0}^{\infty} d s \lambda^{d(1-\alpha)} \tilde{h}_{\alpha}(s, \lambda) \frac{1}{s^{(1+2 \delta) d}} H\left(\frac{\lambda^{1-\alpha} \mathbf{x}}{s^{1+\delta}}, \frac{\mathbf{u}}{s^{\delta}}\right)
$$

Let us consider the case of the simple diffusion operator $L=\Delta . f_{1}(\mathbf{u}, \mathbf{z}, s)$ is then explicitly given as:

$$
f_{1}(\mathbf{u}, \mathbf{z}, s)=\left(\frac{\sqrt{3}}{2 \pi s^{2}}\right)^{d} \exp \left\{-\frac{\mathbf{u}^{2}}{s}+\frac{3 \mathbf{z} \cdot \mathbf{u}}{s^{2}}-\frac{3 \mathbf{z}^{2}}{s^{3}}\right\} \quad .
$$

This pdf exhibits scaling behaviour of the form (41) with $\delta=1 / 2$.

We have not determined the Laplace inversion of this formula. However, we shall obtain the probability distributions for the velocity $\mathbf{u}$ and the spatial variable $\mathbf{x}$. 


\section{The probability distribution $G(\mathrm{x}, t)$}

We shall start with the probability distribution $G(\mathbf{u}, t)$ for the velocity $\mathbf{u}$ which is obtained by an integration with respect to the spatial variable $\mathbf{x}$ :

$$
G(\mathbf{u}, t)=\int d \mathbf{x} f(\mathbf{u}, \mathbf{x}, t)
$$

In Laplace space, we obtain

$$
\tilde{G}(\mathbf{u}, \lambda)=\int_{0}^{\infty} d s \lambda^{\alpha-1} e^{-\lambda^{\alpha} s} G_{1}(\mathbf{u}, s)
$$

which, by Laplace inversion, yields

$$
G(\mathbf{u}, t)=\int_{0}^{\infty} d s h_{\alpha}(s, t) G_{1}(\mathbf{u}, s)
$$

If we assume that the function $G_{1}(\mathbf{u}, s)$ exhibits scaling behaviour,

$$
G_{1}(\mathbf{u}, s)=\frac{1}{s^{d \delta}} g\left(\frac{\mathbf{u}}{s^{\delta}}\right)
$$

the result may be reexpressed in a form which just evidences this scaling behaviour

$$
G(\mathbf{u}, t)=\int d \sigma h_{\alpha}(\sigma) \frac{1}{t^{d \delta \alpha}} g\left(\frac{\mathbf{u}}{t^{\delta \alpha}} \sigma^{\alpha \delta}\right)=\frac{1}{t^{d \delta \alpha}} \tilde{g}\left(\frac{\mathbf{u}}{t^{\delta \alpha}}\right)
$$

Let us consider the case for $L=\Delta_{u}$. Here, $\delta=\frac{1}{2}$ :

$$
G(\mathbf{u}, t)=\int d s \frac{1}{\alpha} \frac{t}{s^{1+1 / \alpha}} L_{\alpha}\left(\frac{t}{s^{1 / \alpha}}\right) \frac{1}{(\sqrt{4 \pi s})^{d}} e^{-\frac{\mathbf{u}^{2}}{4 s}} \quad .
$$

This may also be represented according to

$$
G(\mathbf{u}, t)=\int d \sigma L_{\alpha}(\sigma) \frac{\sigma^{d \alpha / 2}}{\left(\sqrt{4 \pi t^{\alpha}}\right)^{3}} e^{-\frac{\mathbf{u}^{2}}{4 t^{\alpha}} \sigma^{\alpha}} \quad .
$$

Formula (48) yields an interesting structure. First of all, it represents a probability distribution as a superposition of pdf's $G_{1}(\mathbf{x}, s)$ with different variances. Such a representation has been introduced by Castain et al. [12] in a description of the intermittent behaviour of the Eulerian velocity increment pdf. Second, scaling behaviour $<\mathbf{u}^{2}(t)>\sim t$ is quite often related to a random walk behaviour in velocity space. However, the formula (48) shows that such scaling behaviour may also arise for the cases [8] where the scaling indices $\alpha, \delta$ are related by

$$
\delta \alpha=\frac{1}{2}
$$

This e.g. happens for the rotationally symmetric solutions of the diffusion operator

$$
L\left(\mathbf{u}, \nabla_{u}\right)=\nabla\left(\mathbf{u}^{2}\right)^{(1-\alpha)} \nabla
$$

Here, the pdf $G(\mathbf{u}, s)$ is given by

$$
G(\mathbf{u}, s)=N(\alpha) \frac{1}{s^{\frac{d}{2 \alpha}}} e^{-\frac{\mathbf{u}^{2 \alpha}}{4 \alpha^{2} s}}
$$

These solutions have been shown to yield accurate reconstructions of the velocity increment pdf for turbulent flows measured in [5] by adjusting the parameter $\alpha$ [8]. For the case of turbulent flows, the determination of the parameter $\alpha$ and especially its time dependence is an open problem. 


\section{The probability distribution $F(\mathbf{x}, t)$}

Let us now turn to the probability distribution $F(\mathbf{x}, t)$ for the position $\mathbf{x}$. This distribution is obtained from the joint pdf $f(\mathbf{u}, \mathbf{x}, t)$ by integration with respect to the velocity variable:

$$
F(\mathbf{x}, t)=\int d \mathbf{u} f(\mathbf{u}, \mathbf{x}, t)
$$

The Laplace transform of this quantity can be determined from (40)

$$
F(\mathbf{x}, \lambda)=\int_{0}^{\infty} d s \lambda^{d(1-\alpha)} h_{\alpha}(s, \lambda) F_{1}\left(\lambda^{1-\alpha} \mathbf{x}, s\right)
$$

If we consider a system exhibiting scaling behaviour,

$$
F_{1}(\mathbf{z}, s)=\frac{1}{s^{d(1+\delta)}} F\left(\frac{\mathbf{z}}{s^{(1+\delta)}}\right)
$$

we may perform the transformation

$$
S=s \lambda^{-(1-\alpha) /(1+\delta)}
$$

leading to

$$
F(\mathbf{x}, \lambda)=\int_{0}^{\infty} d S \lambda^{(1-\alpha) /(1+\delta)} h_{\alpha}\left(S \lambda^{(1-\alpha) /(1+\delta)}, \lambda\right) \frac{1}{S^{d(1+\delta)}} F\left(\frac{\mathbf{x}}{S^{(1+\delta)}}\right)
$$

Laplace inversion yields the probability distribution

$$
F(\mathbf{x}, t)=\int_{0}^{\infty} d S \frac{1}{S^{d(1+\delta)}} F\left(\frac{\mathbf{x}}{S^{(1+\delta)}}\right) h_{\frac{\delta \alpha+1}{\delta+1}}(s, t)
$$

The decisive step thereby, is the possibility to calculate the Laplace inverse of the function

$$
\begin{aligned}
\mathcal{L}^{-1}\left\{\lambda^{(1-\alpha) /(1+\delta)} h_{\alpha}\left(S \lambda^{(1-\alpha) /(1+\delta)}, \lambda\right)\right\} & =\mathcal{L}^{-1}\left\{\lambda^{\frac{\alpha \delta+1}{1+\delta}-1} e^{-\frac{\alpha \delta+1}{1+\delta} S}\right\} \\
& =\frac{\delta+1}{\alpha \delta+1} \frac{t}{s^{1+\frac{1+\delta}{\alpha \delta+1}}} L_{\frac{\alpha \delta+1}{1+\delta}}\left(\frac{t}{s^{\frac{1+\delta}{\alpha \delta+1}}}\right)=h_{\frac{\delta \alpha+1}{\delta+1}}(s, t)
\end{aligned}
$$

We can now explicitly state the result for the diffusion operator $L=\Delta_{u}$ :

$$
F(\mathbf{x}, t)=\int_{0}^{\infty} d S e^{-\frac{3 \mathbf{x}^{2}}{4 S^{3}}}\left(\sqrt{\frac{3}{4 \pi S^{3}}}\right)^{d} \frac{3}{2+\alpha} L_{(2+\alpha) / 3}\left(\frac{t}{S^{3 /(2+\alpha)}}\right) \frac{t}{S^{1+3 /(2+\alpha)}}
$$

One observes that the limiting case $\alpha=1$ leads to pdf of the Obukhov model. Furthermore, it is evident that the probability distribution of the spatial variable $\mathbf{x}$ is given by an expression similar to the one obtained for the velocity variable in form of an integral transform:

$$
F(\mathbf{x}, t)=\int d \sigma L_{(2+\alpha) / 3}(\sigma)\left(\sqrt{\frac{3}{4 \pi t^{2+\alpha}}}\right)^{d} \sigma^{3(2+\alpha) / 2} e^{-\frac{3 \mathbf{x}^{2}}{4 t^{2+\alpha}} \sigma^{2+\alpha}} \quad .
$$

This expression has to be compared with the one obtained for the pdf of the velocity (50).

We mention that the solution (58) can be related to a continuous time random walk for the position increment $\mathbf{x}(t)$ of a Lagrangian particle. Continuous time random walks for the description of the distance between two Lagrangian variables in connection with Richardson's law have been discussed by Shlesinger et al. [17].

\section{E. Moments of the generalized Obukhov-model}

Starting from eq.400 we can derive a general relation for moments of arbitrary order of the joint probability distribution $f(\mathbf{u}, \mathbf{x}, t)$.

Single-time moments $<x_{i}^{n}(t) u_{i}^{m}(t)>$ are defined as follows:

$$
<x_{i}^{n}(t) u_{i}^{m}(t)>=\int_{-\infty}^{\infty} d \mathbf{u} \int_{-\infty}^{\infty} d \mathbf{x} x_{i}^{n} u_{i}^{m} f(\mathbf{u}, \mathbf{x}, t)
$$


Therefore we obtain in Laplace-space with the help of eq.(32) and eq.(30):

$$
\begin{aligned}
\mathcal{L}\left\{<x_{i}^{n}(t) u_{i}^{m}(t)>\right\} & =\int_{-\infty}^{\infty} d \mathbf{u} \int_{-\infty}^{\infty} d \mathbf{z} \int_{0}^{\infty} d s \delta\left(\mathbf{x}-\lambda^{\alpha-1} \mathbf{z}\right) \tilde{h}_{\alpha}(s, \lambda) x_{i}^{n} u_{i}^{m} f_{1}(\mathbf{u}, \mathbf{z}, s) \\
& =\lambda^{(\alpha-1) n} \int_{0}^{\infty} d s \tilde{h}_{\alpha}(s, \lambda) \int_{-\infty}^{\infty} d \mathbf{u} \int_{-\infty}^{\infty} d \mathbf{z} z_{i}^{n} u_{i}^{m} f_{1}(\mathbf{u}, \mathbf{z}, s)
\end{aligned}
$$

In the last step the integration over the $\delta$-function has been performed. Here we recognize that simply the moments of the ordinary Obukhov-model appear: $\int d \mathbf{u} \int d \mathbf{z} u_{i}^{m} z_{i}^{n} f_{1}(\mathbf{u}, \mathbf{z}, s)=<z_{i}^{n}(s) u_{i}^{m}(s)>$. As as consequence the inverse Laplace-transform leads to a general expression for the moments of the generalized model:

$$
<x_{i}^{n}(t) u_{i}^{m}(t)>={ }_{0} D_{t}^{(\alpha-1) n} \int_{0}^{\infty} d s h_{\alpha}(s, t)<z_{i}^{n}(s) u_{i}^{m}(s)>
$$

Thus the moments $<x_{i}^{n}(t) u_{i}^{m}(t)>$ can be determined from the moments of the ordinary model $<z_{i}^{n}(s) u_{i}^{m}(s)>$ by an inverse Lévy-transform and fractional integration with respect to $t$. Since $<z_{i}^{n}(s) u_{i}^{m}(s)>$ take the form of simple polynomials in $s$ this results in fractional integration of single-time moments of the inverse Lévy-stable process [9]. These calculations can always be performed in an elementary manner.

For example, by applying eq.(65) to the second order moments for the case of $L=\Delta_{u}$ we obtain:

$$
\begin{aligned}
& <\mathbf{u}^{2}(s)>=2 s \quad \rightarrow \quad<\mathbf{u}^{2}(t)>=\frac{2}{\Gamma(\alpha+1)} t^{\alpha}, \\
& <\mathbf{z}^{2}(s)>=\frac{2}{3} s^{3} \quad \rightarrow \quad<\mathbf{x}^{2}(t)>=\frac{4}{\Gamma(\alpha+3)} t^{\alpha+2}, \\
& <\mathbf{z}(s) \mathbf{u}(s)>=s^{2} \quad \rightarrow \quad<\mathbf{x}(t) \mathbf{u}(t)>=\frac{2}{\Gamma(\alpha+2)} t^{\alpha+1} .
\end{aligned}
$$

Clearly, in each case the limit $\alpha \rightarrow 1$ of the Obukhov-model is satisfied. Therefore the generalized Obukhov-model eq. (8) with $L=\Delta_{u}$ is characterized by subdiffusive behaviour of the particle velocity corresponding to the fractional diffusion equation (11) and mean square displacement $\left\langle\mathbf{x}^{2}(t)>\sim t^{\alpha+2}\right.$ of the particle position.

\section{CONCLUSION AND OUTLOOK}

We have introduced a non-Markovian model for the joint position-velocity probability distribution of a random walk particle as a generalization of Obukhov's model. Long-time correlations of the particle velocity are taken into account by fractional time derivatives. As in the limit case of the fractional diffusion equation, a solution can be found as an integral transformation of the Markovian solution. The integral kernel has been obtained by solving a fractional differential equation and yields a complete characterization of the single time statistical properties. As final results we have given analytical expressions for the joint probability distribution and its moments. The generalized Obukhov-model may thus serve as an appropriate model for random walk processes revealing anomalous diffusion in velocity- and position-space.

\section{Acknowledgments}

We gratefully acknowledge support by the Deutsche Forschungsgemeinschaft and wish to thank R. Hillerbrand, O. Kamps and T. D. Frank for helpful discussions.

[1] A. S. Monin, A. M. Yaglom, Statistical Fluid Mechanics: Mechanics of Turbulence, Vol. 1 (MIT Press, Cambridge, MA, 1971); A. S. Monin, A. M. Yaglom, Statistical Fluid Mechanics: Mechanics of Turbulence, Vol. 2 (MIT Press, Cambridge, MA, 1975).

[2] Frisch, Turbulence. The Legacy of Kolmogorov (Cambridge Univ. Press, Cambridge, 1995). 
[3] S. B. Pope, Turbulent flows (Cambridge Univ. Press, 2000).

[4] A.M. Obukhov, Adv. Geophys. 6, 113 (1959).

[5] N. Mordant, P. Metz, O. Michel, J.-F. Pinton, Phys. Rev. Lett. 87, 214501 (2001).

[6] A. La Porta, G. Voth, A. M. Crawford, J. Alexander, E. Bodenschatz, Nature 409, 1017 (2001); A. La Porta, G. Voth, F. Moisy, E. Bodenschatz, Physics of Fluids 12, 1485 (2000).

[7] A.K. Aringazin und M.I. Mazhitov, Int. J. Mod. Phys. B 18, 3095 (2004).

[8] R. Friedrich, Phys. Rev. Lett. 90, 084501, (2003).

[9] A. Baule and R. Friedrich, Phys. Rev. E 71, 026101 (2005).

[10] R. Metzler and J. Klafter, Phys. Rep. 339,1 (2000).

[11] R. Metzler and J. Klafter, J. Phys. A: Math. Gen. 37, R161 (2004).

[12] E. Barkai, R. Metzler, and J. Klafter, Phys. Rev. E 61, 132 (2000).

[13] E. Barkai, Phys. Rev. E 63, 046118 (2001).

[12] B. Castain, Y. Gagne, E. Hopfinger, Physica D 46, 177 (1990).

[15] I. Podlubny, Fractional Differential Equations (Academic Press, San Diego, 1999).

[16] F. John, Partial Differential Equations (Springer, Berlin, 1971).

[17] M.F. Shlesinger, B.J. West, J. Klafter, Phys. Rev. Lett. 58, 1100 (1987). 